\title{
Performance-Based Risk-Sharing Arrangements (PBRSA): Is it a Solution to Increase Bang for the Buck for Pharmaceutical Reimbursement Strategy for Our Nation and Around the World?
}

\author{
Andy Eunwoo Kim ${ }^{1}$. David Hohyun $\mathrm{Choi}^{2} \cdot$ Jongwha Chang ${ }^{3} \cdot$ Sean Hyungwoo Kim ${ }^{1}$ (D)
}

Accepted: 20 September 2020 / Published online: 9 October 2020

(c) Springer Nature Switzerland AG 2020

\begin{abstract}
Due to the risks involved in not achieving desired health outcomes for the dollar spent on drugs, healthcare decision makers, including payers, providers, drug manufacturers, and patients, need a mechanism to share this financial risk among the involved parties. Performance-based risk-sharing arrangements (PBRSAs) are agreements that can potentially reduce the 'drug lag' in which patients wait for an unknown amount of time until a particular drug is covered under their health plan. In addition, PBRSAs can mitigate the risk of investing heavily in drugs that are ineffective or do not deliver good value or "bang for the buck". This review describes and evaluates PBRSAs for drugs in the USA and juxtaposes to other developed nations (i.e. Germany) that adopted PBRSAs in their healthcare model. There are different types of outcomes-based health schemes, namely conditional coverage, which can be further broken down into coverage with evidence development (CED), conditional treatment continuation (CTC), and performance-linked reimbursement, which includes outcomes guarantees. Both CED and CTC are 'conditional' on the collected evidence of the new drug's effectiveness, offering discount only if the drug delivers desirable results. The outcomes guarantee scheme offers discount or even a full refund if the outcome is less than expected, forcing the drug to meet the expected effectiveness. The USA can follow the German reference pricing model in which the assessment of new drugs is centralized and done collectively by representatives from a group of healthcare decision makers. In any shape or form, PBRSA is a clever mechanism to cope with uncertainty if drug price is scaled appropriately based on value.
\end{abstract}

\section{Introduction}

Value in healthcare can be defined in its simplest form as health outcomes achieved per dollar spent on medical care [1]. The idea of value not only focuses on the idea of cost effectiveness but also on the idea of "bang for the buck". The bang for the buck in the US healthcare system with respect to prescription drug expenditure is a growing necessity in terms of: (1) The proportion of prescription drug spending in total health has tripled since the 1980 s and, (2) Prescription

Sean Hyungwoo Kim

skim3@su.edu

1 Shenandoah University Bernard J. Dunn School of Pharmacy, 1775 North Sector Ct, Winchester, VA 22601, USA

2 Torrid Inc., 18501 East San Jose Ave, City of Industry, CA 91748, USA

3 University of Texas at El Paso School of Pharmacy, 500 West University Avenue, El Paso, TX 79968, USA drug spending is expected to increase at a rapid rate of $6.7 \%$ yearly through 2025 [2]. Healthcare decision makers, including payers, providers, and patients (significant cost-sharing for pharmaceuticals in the USA) have placed great importance on understanding the value in order to inform decisions, manage drug spending, and improve the delivery of care [3].

Cost-effectiveness analysis (CEA) is warranted as a tool to quantitatively assess the value provided by pharmaceutical interventions, and thereby assist in decision making [4]. The comparison of cost difference in pharmaceutical interventions divided by difference in effectiveness is a ratio known as incremental cost-effectiveness ratio (ICER). ICER underscores the key principle that effectiveness is measured by clinical outcomes in the form of quality-adjusted life years (QALYs) [4]. QALYs capture the two distinct dimensions of improved clinical effectiveness: increased life-years of survival, and improved quality of life [4]. In particular, as the denominator shrinks toward zero, the ICER rises towards infinity, implying that interventions that produce little to no 


\section{Key Points}

Healthcare decision makers, including payers, providers, drug manufacturers, and patients can benefit from performance-based risk-sharing arrangements (PBRSAs) that employ cost-effectiveness analysis (CEA) to quantitatively assess the value provided by a new health technology and assist decision-making based on that value.

Outcome-based contracting, including PBRSAs, is an attractive way to measure and reduce the risks and uncertainties that come along with the coverage of new drugs without sufficient evidence of its effectiveness and the approval of a new therapy that is potentially promising but very expensive.

Performance-based risk-sharing arrangements will help payers, providers and drug manufacturers to cope with uncertainties regarding the effectiveness of a new drug only if the price of the drug is set appropriately, based on the clinical value received by the involved parties.

improvement in quality-adjusted survival cannot yield an acceptable ICER or bang for the buck [4]. In addition, it is important to note disability-adjusted life years (DALYs) as another metric to calculate ICER. Unlike the QALYs that are more frequently used by decision scientists, health economists and policymakers in high-income countries, DALYs were introduced in the 1993 World Development Report [5]. DALYs estimate the gap between a population's health status and an "ideal" level of health and survival from the following equation: DALYs = YLL (years of the life lost $)+$ YLD (years lived with disability) or the sum of a population's YLL to premature death and the YLD [5]. In comparison to QALYs, DALYs are frequently used by economists, epidemiologists, and policy experts, especially those who work on health issues in low- and middle-income countries, for population health assessments, priority setting and program evaluation [5]. Hence, ICER can be calculated by using either QALYs or DALYs in the denominator of the equation.

From a payer perspective, at the time the reimbursement decision is made, the real value of a drug is uncertain in a real-life clinical setting (drug effectiveness) [6]. This poses a financial risk from a payer perspective since the effectiveness of a drug may be lower than predicted from an efficacy standpoint (i.e. controlled or ideal environment) [7]. This uncertainty in health outcomes can potentially create 'drug lag' for patients until a particular drug is covered under the health plan. Hence, several types of policies, agreements, or arrangements have been designed to mitigate this uncertainty by sharing the financial risk between the payer and the drug manufacturers [6]. Such agreements are called the performance-based risk-sharing arrangements (PBRSAs) or risk-sharing agreements (RSAs). The financial risk sharing may allow novel drugs to be accepted for reimbursement rather early (shorten drug lag) while preventing the waste of resources on drugs that are ineffective or provide less bang for the buck. In this review, we plan to describe and evaluate PBRSAs for drugs in the USA while juxtaposing with developed countries that have adopted PBRSAs for drug reimbursement.

\section{The Decision-Making Process}

Health technology assessment (HTA) is defined as the practice of evaluating new health technologies for the purpose of deciding what an insurance plan will cover [8]. CEA is an important tool to evaluate novel pharmaceuticals as CEA compares the bang for the buck or value of various pharmaceutical competing choices or alternatives. At the time a reimbursement decision is made, the real value of a drug is often uncertain [6].

A nation with a single-payer healthcare system providing universal health coverage to their citizens through general tax revenues such as the UK, has a government agency known as National Institute for Health and Care Excellence (NICE). The NICE conducts HTA to deal with moral hazard problems that arise when people have no sensitivity to healthcare costs. The fundamental health economics tradeoff with novel pharmaceuticals starts from the question of: "Should our plan allow this new pharmaceutical agent to be covered under our health plan?" On the one hand, allowing coverage increases accessibility of pharmaceuticals to patients and providers. However, on the other hand, if the new agent is not cost effective or if the bang for the buck is mediocre, tax revenue will be wasted. A nation with 'free' healthcare coverage through supply-side rationing should be more cognizant of cost as moral hazard can decrease efficiency of healthcare spending. Supply-side rationing may limit access to novel pharmaceuticals by mechanisms such as gatekeeping (i.e. prior authorization) or restricting coverage in the formulary.

What of our nation? The US healthcare delivery relies on private payers for the majority of Americans. Each payer has its unique formulary or list of drugs covered under the plan. The Pharmacy and Therapeutics (P\&T) committee from each payer conducts their own HTA whether to allow coverage of new drugs for their beneficiaries. The US healthcare delivery provides health insurance coverage for elderly citizens via Medicare, similar to the entitlement health insurance plan for Canadians. Although CEA can help to limit moral hazard, this creates a controversy since 
denying coverage on a potential life-saving treatment such as innovative oncology drugs (i.e. last resort therapy option or novel mechanism of action) or conditions that are ongoing (i.e. insurance plan not covering drugs for COVID-19) due to lack of evidence on its effectiveness evidence, may increase statistical death [8]. Statistical death is defined as death of persons who may die because a life-saving drug is unavailable [8]. This important trade-off is deemed illegal in the USA, forbidden by law from using CEA in its coverage decisions for Medicare patients [8]. This implies that cost should not be a consideration so long as an intervention is medically effective [9]. However, private payers still rely on CEA or comparative effectiveness research (CER) to create their own formulary.

\section{The Need for Financial Risk-Sharing Mechanism}

It is widely accepted that randomized clinical trials (RCTs) cannot provide all necessary information about the safe and effective use of drugs at the time they are marketed [10]. In the USA, drugs cannot be marketed for Food \& Drug Administration (FDA) approval without an acceptable safety and efficacy profile from RCTs [11]. Although there are inherent limitations of RCTs during the drug development process, such as having a small sample size that often under-represents vulnerable patient groups and focusing on a short-term efficacy and safety in a controlled environment (ideal setting), FDA will approve a drug if there is substantial evidence of effectiveness for the proposed use and if the benefits outweigh the risks and remaining uncertainties $[10,12]$. Effectiveness takes into account the loss of drug efficacy due to routine clinical practice obstacles such as patient medication adherence rate and subgroup variability of drug response in a population. This implies that effectiveness of a drug may have less than predicted efficacy, creating a financial risk for healthcare payers [6]. This is precisely the fundamental underlying reason why 'drug lag' occurs; drug companies desire to market their drugs as soon as approval occurs and also to increase market share by negotiating with payers to allow coverage. Hence, there is a need for a risksharing mechanism that satisfies both drug manufacturers and payers, precisely via the performance-based schemes or PBRSAs. The manufacturers must guarantee the effectiveness of drugs to some extent because payers will not allow coverage if there is large uncertainty. If many companies have a money-back guarantee program to boost confidence in buyers, then drug manufacturers will need to guarantee the effectiveness of the drug to some extent via health outcome-based agreement in order to compete with rival manufacturers. Such agreements may allow drugs to be accepted for reimbursement relatively early, while preventing the waste of public resources on drugs that are ineffective or do not live up to expectations $[13,14]$.

\section{Performance-Based Schemes Between Payers and Manufacturers}

In 2010, Carlson et al. conducted a systematic review on performance-based or health-outcomes reimbursement schemes delineating non-outcomes-based as well as health outcome reimbursement schemes [14]. The central focus of this review is to only focus on the health outcomes-based schemes because the fundamental point of this agreement between manufacturer and payer stem from those in which the price, level, or nature of reimbursement are tied to future performance measures of clinical or intermediate ends points ultimately related to patient quality/quantity of life [14].

Subcategories of health outcomes-based schemes are defined as follows: (1) conditional coverage: where coverage is granted conditional to the initiation of a program of data collection; (2) performance-linked reimbursement (PLR): where reimbursement level for covered product is based on the measure of clinical outcomes in the "real world"; (3) coverage with evidence development (CED): is a scheme where coverage is conditional on the initiation of a program of data collection that informs the use of the medical product in the payer population; (4) conditional treatment continuation (CTC): is a scheme where payer continues to cover only for individual patients that benefit from the treatment; (5) outcome guarantees: is a scheme where the manufacturer provides rebates, refunds, or price adjustment if their product fails to meet the outcome targets agreed upon [14].

\subsection{Conditional Coverage}

Conditional coverage is a performance-based scheme where coverage is granted conditional to the initiation of a program of data collection that informs the use of the medical product in the payer population [14]. According to Fig. 1, CED creates a middle ground for payer to deal with uncertainty in effectiveness of the drug or medical device [14]. For example, risperidone is a drug classified as an atypical antipsychotic used for patients with schizophrenia. The manufacturer Johnson and Johnson (J\&J) and French Ministry of Health entered into a CED agreement where the French Ministry of Health agreed to cover risperidone at J\&J's asking price conditional upon J\&J's follow-up study of whether the drug helps patients stay on their medication. J\&J will reimburse France's Ministry of Health a portion of the money spent on the drug if the studies show otherwise [14].

Conditional treatment continuation (CTC) is a performance-based scheme where payer continues to cover the cost of the drug or medical device based on short-term treatment 
goals to ensure that only patients that benefit from the treatment will remain on treatment coverage. Multiple manufacturers for Alzheimer's disease drugs entered into a CTC agreement with Agencia Italiana del Farmaco (AIFA) or the Italian Drugs Agency to assess short-term effectiveness of Alzheimer's disease drugs based on the premise that during the first 3 months, drugs will be covered for free by the manufacturers. If the treatment goals are met after 3 months, only those with treatment benefits will be continued on the treatment for 2 years where drug costs are reimbursed by the AIFA [14].

\subsection{Performance-Linked Reimbursement}

Performance-linked reimbursement (PLR) scheme is a commonly utilized method of arrangement when there are multiple competing choices of drugs for the same indication. The idea of value-proposition or bang for the buck story around their new products needs to be elucidated, not only in terms of clinical benefit and safety, but also on secondary elements such as convenience that leads to increased medication adherence (i.e. convenient oral route of administration rather than subcutaneous injection for anti-diabetic drugs, oral route of administration preferred over intraurethral for the treatment for erectile dysfunction).

PLR schemes are likely to arise when a manufacturer has sufficient confidence in their product claims and that they are willing to accept a lower reimbursement level if it under-performs [14]. For instance, the Deutsche Angesteilten-Krankenkasse or German Sickness Fund entered into an arrangement with Novartis on an immunosuppressant cyclosporine, mycophenolic acid, and everolimus for patients with kidney transplant to prevent autoimmune response. The manufacturer agreed to refund the cost of the drug if a patient loses his/her donor kidney [14]. Without confidence in their product, manufacturers would not enter into such an agreement.

PLR schemes are also used as a mechanism for manufacturers to increase market share of their product. The German Sickness Fund entered into an agreement with a manufacturer, Novartis, for osteoporosis drug-zoledronic acid. Novartis will cover the drug costs of any patient who experiences a fracture within 1 year of being treated with zoledronic acid. In return, the German Sickness Fund agrees to shift the treatment of its patients to zoledronic acid while Novartis increases their product share in the osteoporosis market [14]. However, this contractual agreement with

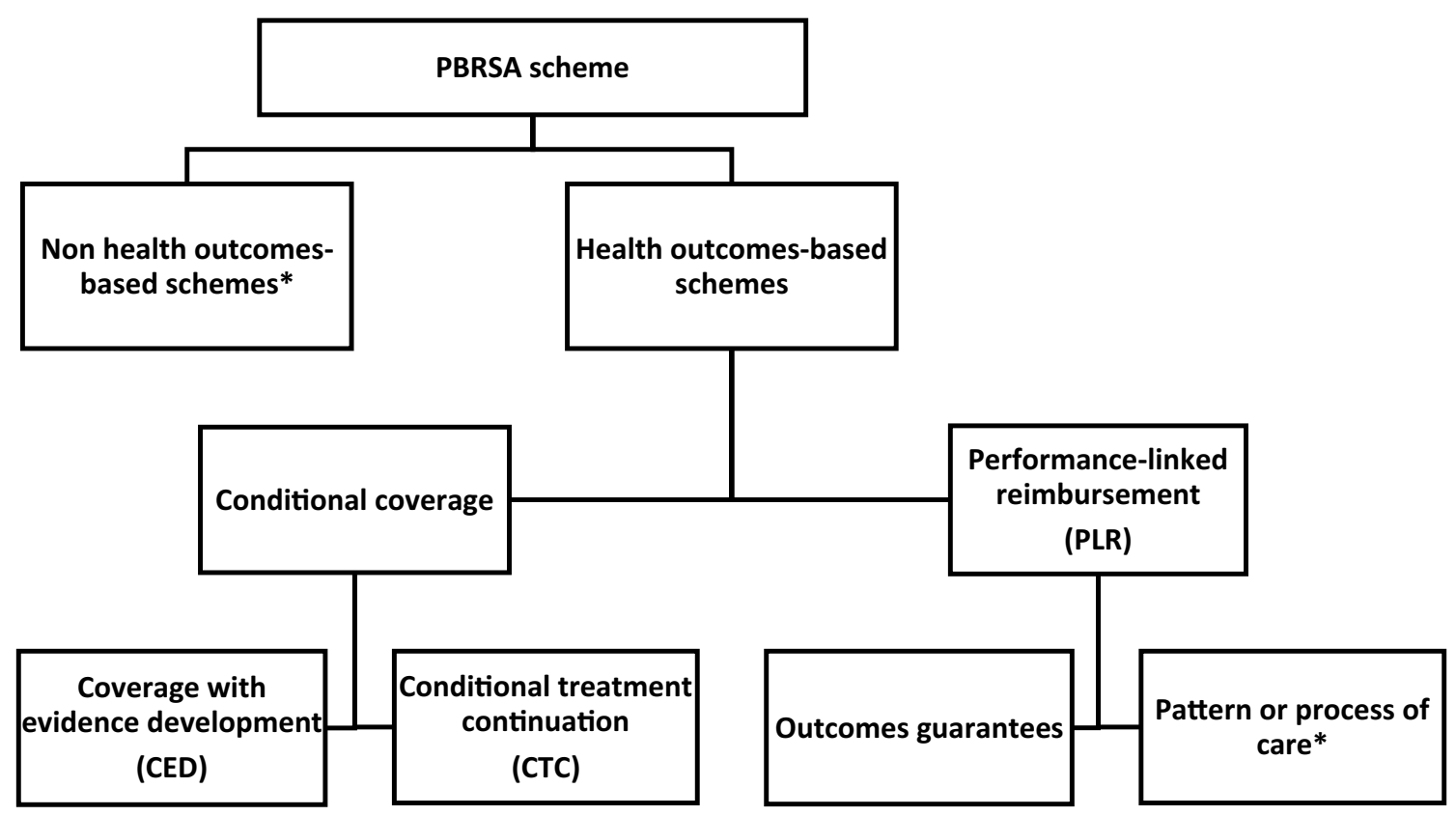

Fig. 1 Performance-based risk-sharing arrangement (PBRSA) scheme. Subcategories of health outcomes-based schemes are defined as follows: (1) conditional coverage: coverage is granted conditional on the initiation of a program of data collection; (2) performancelinked reimbursement (PLR): reimbursement level for covered product is based on the measure of clinical outcomes in the "real world"; (3) coverage with evidence development (CED): scheme where coverage is conditional on the initiation of a program of data collection that informs the use of the medical product in the payer population; (6) conditional treatment continuation (CTC): scheme where payer continues to cover only for individual patients that benefit from the treatment; (7) outcome guarantees: scheme where the manufacturer provides rebates, refunds, or price adjustment if their product fails to meet the agreed upon outcome targets [14]. *Non health outcomesbased schemes and pattern or process of care are outside the scope of this review 
German Sickness Fund was withdrawn due to challenges in measuring patient health outcomes.

\section{PBRSAs for Pharmaceutical Products in the USA: Where Are We Going Next?}

In 2017, Yu et al. conducted a systematic review identifying 26 US-specific PBRSAs for drugs announced or initiated from 1997-2017 [2]. Over half of the PBRSAs identified involved drugs with cardiometabolic indications. Given the magnitude of the American population affected by diabetes and coronary heart diseases (30.3 million and 15.4 million, respectively), payers will increase focus on managing costs of drugs with potential for high-budget impact $[2,15,16]$. CEA becomes even more important for these conditions since saving a dime or even a penny per tablet could be substantial; chronic medications to lower low-density lipoprotein cholesterol (LDL-C) or blood pressure are to be taken by patients over many decades. It is important to note that lowering LDL-C or blood pressure levels is not the final cardiovascular health consequence; these proxy indicators are known as intermediate cardiovascular health consequences. A relatively new class of cholesterol-lowering agent called protein convertase subtilisin/kexin type 9 (PCSK9) inhibitors (Trade name: Repatha ${ }^{\circledR}$, Generic name: evolocumab) exemplify a need to adopt the PBRSAs in the pharmaceutical market - a new therapy that is potentially promising but very expensive. The idea of bang for the buck and uncertainty of its effectiveness can have a huge budget impact on payers [4]. Imagine that a single new agent to reduce cholesterol levels with many competing therapeutic alternatives can increase every American's health insurance premium by $\$ 124$ per year [17]. What would you do if you were the chair of the P\&T committee? What is the justification for allowing this cholesterol-lowering drug to be in the formulary?

Payers can align payments for drugs with "value" they create based on their willingness-to-pay, while drug manufacturers in turn will receive incentives to continue developing new treatments [2]. Amgen's PCSK-9 inhibitor, evolocumab, entered into a PBRSA in 2016 with Cigna. Amgen would further discount the cost of evolocumab if Cigna's customers were not able to reduce LDL-C levels to at least levels experienced in clinical trials. These risksharing arrangements could have a huge impact on millions of patients who have been treated with PCSK9 inhibitors for decades at a cost of $\$ 14,000$ per year [4], an amount that has been an incredibly expensive alternative for most patients, Amgen decided to reduce the out-of-pocket costs for Medicare Part D beneficiaries in 2018. Amgen has committed to distribute Repatha ${ }^{\circledR}$ exclusively at the $60 \%$ lower list price of $\$ 5850$ per year (approximately $50 \%$ of Part D beneficiaries but will get it at this price) [18]. However, Repatha ${ }^{\circledR}$ is still expensive in the realm of cost-effective alternatives among lipid-lowering agents. Can Repatha ${ }^{\circledR}$ compete with the $<\$ 10 /$ month generic lipid-lowering agents in terms of cost effectiveness? According to the Avalere's analysis in 2019, the majority of patients without low income subsidies under Part D will continue to face high monthly costs and almost $50 \%$ of Part D beneficiaries are expected to face cost sharing of $\$ 50$ or more to access PCSK9 inhibitors in 2020 [19]. As mentioned previously, there are two ways to increase the value-proposition through ICER: Decrease the drug cost difference or increase the difference in its effectiveness. Which route is more feasible from the manufacturer's standpoint?

PBRSAs can provide further discounts on the cost of the product and enable those patients who can most benefit from the drug to receive it while continuing to encourage utilization of lower-cost statins for the majority of patients. In 2016, Amgen entered into a PBRSA with Harvard Pilgrim to provide enhanced discount of the cost of evolocumab if the reduction in LDL levels for the Harvard Pilgrim members is less than that of clinical trials. The contract included additional discounts if the utilization of the drug exceeds agreed levels. Amgen also agreed to an adherence provision that conditions discounts on patients reliably taking the drug [2]. Harvard Pilgrim has entered into a second contract with Amgen in 2017 that guarantees the health plan and its members will receive a full refund of drug costs if a member is hospitalized for a myocardial infarction or stroke after taking evolocumab for 6 months or more and maintaining an appropriate level of drug compliance [20].

In 2018, Pearson, Nichols et al. pointed out a major distinction between performance-based reimbursement schemes between payer and drug manufacturer versus true value-based drug pricing. Outcome-based contracting, including PBRSA, offers an attractive way to link pricing to clinical value with great potential, but only if pricing can be scaled appropriately with clinical value [21]. Two competing drugs that lower LDL-C levels with more than 100 times the difference in cost is an epitome of how drug price was not scaled appropriately per bang for the buck in the USA. Hence, is PBRSA a marketing gimmick for drug manufacturers to justify their high price if drug price is not scaled appropriately? Alternatively, this agreement may even risk diverting attention from the larger goal of linking drug prices in a reasonable and proportional way to their relative added benefits for patients [21]. The complex nature of payers' willingness to pay for incremental drug therapeutic benefit conglomerated with manufacturers' drug pricing practice based on price discrimination drives the need for CER analyzing three dimensions of pharmaceuticals: Benefits, risks, and costs are all critical components of CER. 


\section{PBRSAs for Pharmaceutical Products in the German Sickness Funds: Internal Reference Pricing}

In the USA, the level of cost-sharing and resulting financial burden on patients is high [22]. US payers often impose modest copayments on low-cost drugs with many direct substitutes but onerous coinsurance on high-cost drugs (i.e. specialty drugs) with few substitutes [22]. The German Sickness Funds also has a modest level of cost-sharing between payer and patients to influence consumer choices for drugs with therapeutically equivalent alternatives [22]. However, unlike the USA, German insurance design for pharmaceutical reimbursement is built on internal reference pricing in a centralized fashion by the Federal Joint Committee (GBA) and Institute for Quality and Efficiency in Healthcare (IQWiG) rather than each payer's P\&T committee. The term "centralized approach" refers to the notion that the entire German Sickness Funds will have the purchasing power to build a national formulary (a.k.a. collective contractual system) unlike the USA where each payer (i.e. private insurer or Centers for Medicare and Medicaid) has to negotiate with manufacturers for pricing. Hence, clinical assessment of each new drug is conducted in a centralized fashion via internal reference pricing scheme (GBA sets up the reference pricing groups) in addition to the drug price negotiation by the German umbrella organization (GKV-SV or The National Association of Statutory Health Insurance Funds) of health insurers [22]. Although manufacturers are free to set the prices of their products, insurers will not pay more for a new drug than its comparators unless it offers an additional clinical benefit (based on GBA internal reference pricing) [22]. Reference pricing will drive PBRSA in German healthcare system to some extent between payer and manufacturers but there are shortcomings. The German pharmaceutical reimbursement mechanism does not use real-life clinical performance to value pharmaceuticals.

Suppose a new drug was approved by the European Medicines Agency (EMA is a European equivalent of the US FDA). During the first year of launch of the new drug, comparative clinical safety and efficacy assessment is conducted by the GBA for internal reference pricing [22]. The GBA is an organization that assesses the comparative clinical performance of new drugs consisting of representatives of the national insurance, physician, and hospital organizations [22]. GBA makes important decisions regarding assessment of each drug's incremental benefit with input from the IQWiG, the pharmaceutical manufacturer, relevant medical associations, patient advocacy organizations and other interested entities [22]. First, GBA decides which drug will be used as the comparator (reference drug) against which the new product is to be assessed. Second, GBA chooses the metrics that will assess the new drug's benefit, requiring pharmaceutical firms to provide metrics that EMA does not require, quality-of-life indicators such as change in pain and nausea [22]. GBA delegates the clinical evaluation of the new drug to IQWiG, which bases its evaluations on dossiers submitted by manufacturers. Assessment of the mandatory dossier is done by the IQWiG on the basis of published data, ideally from high-level evidence RCTs. Appraisal is done after a certain period by the GBA, while price negotiations are to be done by GKV-SV. If the new drug has no additional therapeutic benefit, it is transferred to the reference pricing scheme in order to evaluate safety profile (i.e. detrimental consequences) relative to the alternatives at a similar price level. At this stage, manufacturers sometimes decide to withdraw their product from the market. In addition, systematic review of the incremental benefit versus risks of the drug, results from the clinical trials for initial market authorization by the EMA, and reports by technology assessment agencies in other nations could all be utilized for official assessment [22]. Lastly, the GBA makes its official assessment of each drug's contribution based on IQWiG study. The final assessment of the drug's benefit is utilized by the German GKV-SV.

Could the USA employ this centralized approach like Germany, despite complex multiple payer healthcare systems? The German and US employer-sponsored health insurance share similarities where both employer and employee contribute to financing healthcare and drug costs. Hence, although there may be subtle differences between the two nations' healthcare delivery, the grand scheme of centralized pharmaceutical reimbursement strategy with internal reference pricing and collective contractual system could be introduced in the USA as long as all payers (including both private and public) can find the middle ground. However, as aforementioned, lack of real-life effectiveness data to scale the value of pharmaceuticals is a shortcoming that needs to be addressed in order to use PBRSA for both nations.

\section{Conclusion}

The fundamental trade-off with newly approved pharmaceuticals is to deal with statistical death versus identifiable death. Should a payer value statistical life or death that could have been prevented by allowing new drug coverage (also known as Type II error)? When a payer delays coverage, beneficiaries who cannot afford high drug prices will have no access to the therapeutic option. Alternatively, should a payer value identifiable death or death that could have been prevented by allocating healthcare dollars to more cost-effective interventions that decrease the morbidity and mortality of their beneficiaries (also known as Type I error)? Certainly, the PBRSA is a clever mechanism to help payers, 
providers, and drug manufacturers to cope with uncertainty in pharmaceuticals, only if drug price is scaled appropriately based on value or bang for the buck.

Acknowledgements We are grateful to the authors of the contributing works and Jaehee Lee and newborn Angelina Taeri Kim for all the social support.

\section{Declarations}

Funding No funding was obtained for this study.

Conflicts of Interest Andy Eunwoo Kim, David Hohyun Choi, Jongwha Chang and Sean Hyungwoo Kim report no conflict of interest in this work.

Ethics Approval Not applicable in this review.

Ethics Committee or Institutional Review Board Not applicable in this review.

Informed Consent Not applicable in this review.

Availability of Data and Material Not applicable in this review.

Code Availability Not applicable in this review.

Author's Contributions Andy Eunwoo Kim and David Hohyun Choi were responsible for idea and draft writing for the manuscript. Jongwha Chang was responsible for formatting and editing the overall manuscript. Sean Hyungwoo Kim was responsible for idea, draft writing, and overall supervision of the editing, manuscript writing, as well as all the support for literature search and providing resources.

\section{References}

1. Porter ME. What is value in health care? N Engl $\mathrm{J}$ Med. 2010;363(26):2477-81.

2. Yu JS, Chin L, Oh J, Farias J. Performance-based risk-sharing arrangements for pharmaceutical products in the United States: a systematic review. J Manag Care Spec Pharm. 2017;23(10):1028-40.

3. Sorenson C. Use of comparative effectiveness research in drug coverage and pricing decisions: a six-country comparison. Issue Brief (Commonw Fund). 2010;91:1-14.

4. Hlatky MA, Kazi DS. Economics of PCSK9 inhibitors. J Am Coll Cardiol. 2017;70:2677-87.

5. Chen A, Jacobsen KH, Deshmukh AA, et al. The evolution of the disability-adjusted life year (DALY). Socio-Econ Plan Sci. 2015. https://doi.org/10.1016/j.seps.2014.12.002.

6. Holleman MS, Uyl-de Groot CA, Goodall S, Linden NVD. Determining the comparative value of pharmaceutical risk-sharing policies in non-small cell lung cancer using real-world data. Value Health. 2019;22(3):322-31.
7. Sanders GD, Neumann PJ, Basu A, et al. Recommendations for conduct, methodological practices, and reporting of cost-effectiveness analyses: second panel on cost-effectiveness in health and medicine. JAMA. 2016;316:1093-103.

8. Bhattacharya J, Hyde T, Tu P. Health economics. 2013th ed. London: Palgrave Macmillan; 2014.

9. Neuman PJ, Rosen AB, Weinstein MC. Medicare and cost-effectiveness analysis. N Engl J Med. 2005;53(14):1516-22.

10. Schneeweiss S, Avorn J. A review of uses of health care utilization databases for epidemiologic research on therapeutics. J Clin Epidemiol. 2005;58(4):323-37.

11. Milestones in U.S. food and drug law history. U.S. Food and Drug Administration. 2009. https://www.fda.gov/about-fda/fdas-evolv ing-regulatory-powers/milestones-us-food-and-drug-law-history. Accessed 30 Mar 2020.

12. Gassman AL, Nguyen CP, Joffe HV. FDA regulation of prescription drugs. N Engl J Med. 2017;376(7):674-82.

13. Walker S, Sculpher M, Claxton K, Paler S. Coverage with evidence development, only in research, risk sharing, or patient access scheme? A framework for coverage decisions. Value Health. 2012;15:570-9.

14. Carlson JJ, Sullivan SD, Garrison LP, et al. Linking payment to health outcomes: a taxonomy and examination of performancebased reimbursement schemes between healthcare payers and manufacturers. Health Policy. 2010;96:179-90.

15. National diabetes statistics report, 2020. Centers for Disease Control and Prevention. 2020. https://www.cdc.gov/diabetes/pdfs/ data/statistics/national-diabetes-statistics-report.pdf. Accessed 12 Apr 2020.

16. Go AS, Mozaffarian D, Roger VL, Benjamin EJ, Berry JD, Blaha MJ, et al. Heart disease and stroke statistics-2014 update. Circulation. 2014;129(3):e28-292.

17. Schulman KA, Balu S, Reed SD. Specialty pharmaceuticals for hyperlipidemia-impact on insurance premiums. N Engl J Med. 2015;373:1591-3.

18. Akopyan J, Hawkins T, Sood A. Amgen to make Repatha ${ }^{\circledR}$ (evolocumab) available exclusively at its lower list price option in 2020. In: Press release. Amgen. 2019. https://www.amgen.com/ media/news-releases/2019/10/amgen-to-make-repatha-evolo cumab-available-exclusively-at-its-lower-list-price-option-in2020/. Accessed 2 Sept 2020.

19. Sullivan M, Whittam D, Olaughlin C. Affordable patient access to PCSK9 inhibitor remains challenging across part D plans in 2020. 2019. https://avalere.com/insights/affordable-patient-acces s-to-pcsk9-inhibitors-remains-challenging-across-part-d-plans-in2020. Accessed 2 Sept 2020.

20. Fallon J. Harvard pilgrim signs second groundbreaking contract with Amgen for Repatha. Harvard Pilgrim Healthcare. 2017. https://www.harvardpilgrim.org/public/news-detail?nt=HPH News_C\&nid=1471912937208. Accessed 30 Mar 2020.

21. Pearson S, Nichols L, Chandra A. Policy strategies for aligning price and value for brand-name pharmaceuticals. Health Aff. 2018. https://doi.org/10.1377/hpb20180216.92303.

22. Robinson JC, Panteli D, Ex P. Reference pricing in Germany: implications for U.S. pharmaceutical purchasing. Issue Brief (Commonw Fund). 2019;2019:1-8. 